\title{
Early diagnosis of exudates information and deduction of diabetic retinopathy using level set segmentation based on multi wave propagation.
}

\author{
S. Vijayalakshmi ${ }^{1}$, and P. Sivaprakasam ${ }^{2}$ \\ Research Scholar of Karpagarm University, Coimbatore, \\ Lecturer, Department of M.C.A Park College of Engineering and Technology Coimbatore-641059 \\ ${ }_{2}$ Associate Professor Sri Vasavi College Erode-638 316
}

\begin{abstract}
Diabetic retina is a result of increase in the blood glucose level leading to damage of retinal blood vessels causing temporary or permanent blindness. The increase in number of cases of diabetic retina emphasizes the need of early detection before it reaches the severe stage. The diabetic retina unless other type of medical scans has multiple clots of bloods inside the vessels. Hence single curve is not sufficient to detect the multiple clots. A methodology is proposed here for the detection of multiple clots for solving a non unique solution level set segmentation.
\end{abstract}

\section{INTRODUCTION}

Segmentation ${ }^{[1]}$ is defined as the portioning of the image based on certain criterions as level of intensity, spread of the RGB colour index, inhomogeneity in brightness etc. The objective behind the implementation of the of level set algorithm is based on the active contour methods abilities ${ }^{[2]}$. The level set segmentation problem reduces to findings of the curves to enclose the region of interest ${ }^{[3]}$. Hence the curves can be modeled directly using the control points.

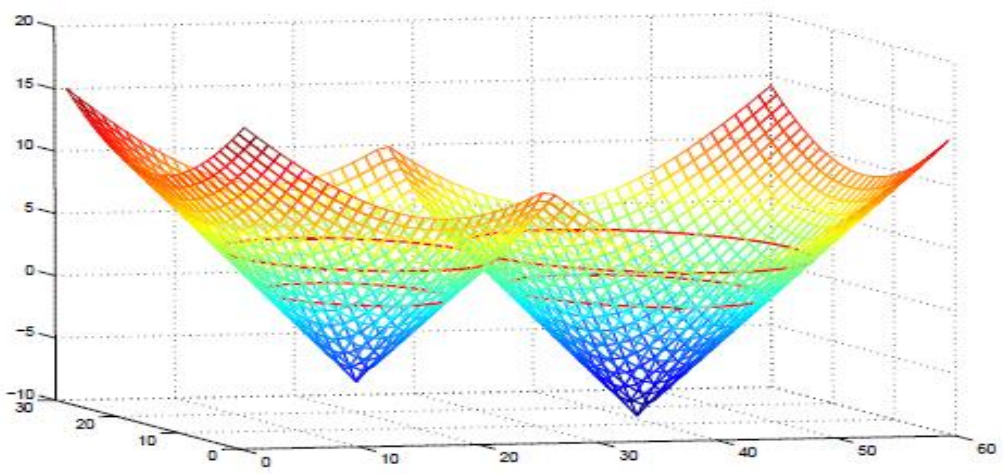

Figure 1: Figure demonstrating the embedding of one closed contour relating to the other two closed contour of the same level.

\section{Simple formulation of the Level set algorithm}

The problem can be formulated using an active contour propagation method. Taking into account the segmentation boundary as a part of a surface where the contour level is zero. This is also known as zero level set.

Let $\Phi(x, t)= \pm d$

Here $\mathrm{x}$ is the position in the start or originating domain. $\mathrm{T}$ is the time and $\mathrm{d}$ is the distance between position $\mathrm{x}$ and the zero level set. $\mathrm{d}$ is positive, if $\mathrm{x}$ is outside zero, else $\mathrm{d}$ is negative. Curve of interest originates from $=0$. which is marked.

To evolve $\Phi$ over time, use chain rule

$\Phi_{t}+\Phi x_{t}+\Phi_{y} y_{t}=0 .$. (2)

$\Phi_{t}+\left(x_{t} y_{t}\right) \nabla \Phi=0$. (3) 
Let $(\mathrm{xt}, \mathrm{yt})=\mathrm{n}+\mathrm{s}$

Where $\mathrm{n}$ is vector normal to the front at point $\mathrm{x}$ and $\mathrm{s}$ is some arbitrary vector. Since $\mathrm{n}$ and $\mathrm{s}$ are defined over the entire domain of $\mathrm{x}$, they are actually vector fields.

$$
\begin{aligned}
& \Phi_{t}+(n+s) \cdot \nabla \Phi=0 \ldots(4) \\
& \Phi_{t}+n \cdot \nabla \Phi+s \cdot \nabla \Phi=0 . .(5) \\
& \Phi_{t}+V n \cdot|\nabla \Phi|+s \cdot \nabla \Phi=0 . .(6)
\end{aligned}
$$

Here $\mathrm{Vn}$ is some scalar.

Values $\mathrm{Vn}$ and s can be viewed as two independent forces that evolve from the surface. The value of Vn will control how fast the surface will move in the normal direction. S dictates both direction and speed of evolution. The partial differential equations can then be solved when provided initial condition.

$\Phi\left(\mathrm{x}_{y} \mathrm{t}=0\right)$. ( ( $)$

Hence the problem can be defined as the initial value problem.

Equation 1 can be solved by using a finite difference on a discrete spatial grid in domain of $\mathrm{x}$.

$\operatorname{Let}^{\Phi}(\mathrm{x}, \mathrm{n} \Delta \mathrm{t})=\Phi_{\mathrm{x}^{\mathrm{x}}}^{\mathrm{n}}$ (8)

$$
\begin{aligned}
& \frac{\Phi_{i j}^{n+1}-\Phi_{i j}^{n}}{\Delta \mathrm{t}}+V_{n}\left|\nabla \Phi_{i j}^{n}\right|+S_{i j} . \Phi_{i j}^{n}=0 . .(9) \\
& \Phi_{i j}^{n+1}=\Phi_{i j}^{n}-\left[V_{n}\left|\nabla \Phi_{i j}^{n}\right|-S_{i j} . \Phi_{i j}^{n}\right] . \Delta \mathrm{t} \ldots(10)
\end{aligned}
$$

Curve speed and vector fields

$\mathrm{Vn}$ and $\mathrm{S}$ can be implemented as

$\Delta I^{G}=\left(I_{z,}^{G}, I_{y}^{G}\right) .(11)$

$$
\begin{gathered}
V_{n}=\exp \left(-\propto\left|\nabla I^{G}\right|\right) . .(12) \\
S=\beta \Delta\left|\nabla I^{G}\right| . .(13)
\end{gathered}
$$

Ig is the input image blurred with a constant Gaussian filter. $\propto$ is a constant for correcting the how fast Vn approaches 0 , when edge encounters image and $\beta$ is a constant for controlling the strength of $\mathrm{S}$. The parameters for the Gaussian filter are set at the user end. When the input image has small gradient the $\mathrm{Vn}$ approaches 1 and $\mathrm{S}$ approaches zero. However when an edge is encountered $\mathrm{Vn}$ approaches 0 and $\mathrm{s}$ will point towards the edge. A larger $\propto$ will let the Vn approach 0 at faster rate. On the other hand the $\sigma$ the Gaussian filter coefficient preserves more details of the image however the edges will be blurred. For default contraction, $\mathrm{Vn}$ is multiplied by -1 . The values are calculated over central difference.

$$
\begin{aligned}
\left(I_{x}^{G}\right)_{i j} & =\frac{I_{i+1, j}^{G}-I_{i-1, j}^{G}}{2} \ldots(14) \\
\left(I_{y}^{G}\right)_{i j} & =\frac{I_{i j+1}^{G}-I_{i, j-1}^{G}}{2} \ldots(15) \\
\left|\nabla I^{G}\right| & =\sqrt{\left(I_{z}^{G}\right)_{i j}-\left(I_{y}^{G}\right)_{i j} \ldots(16)} \\
V_{n} & =\exp \left(-\propto\left|\nabla I^{G}\right|\right)
\end{aligned}
$$

$\left(\left|\nabla I_{z}^{G}\right|\right)_{i, j}=\frac{\left|\nabla I^{G}\right|_{i+1, j}-\left|\nabla I^{G}\right|_{i-1, j}}{2} \ldots$

$\left(\left|\nabla I_{Y}^{G}\right|\right)_{i j,}=\frac{\left|\nabla I^{G}\right|_{i, j+1}-\left|\nabla I^{G}\right|_{i, j-1}}{2} \ldots$

$$
S_{i, j}=\beta\left(\left.C|C \quad| \nabla I^{G}\right|_{x}\right)_{i j}\left(\left.|C| \nabla I^{G}\right|_{y}\right)_{i j} \ldots
$$




\section{Entropy Condition}

The degree of randomness can be specified by considering

$u_{t}(x, t)=-a(x) u_{x} x(t) \ldots(21)$

$\frac{d t}{d x}=\left\{\begin{array}{rl}-1 & x<0 \\ 1 & x>0\end{array}\right.$

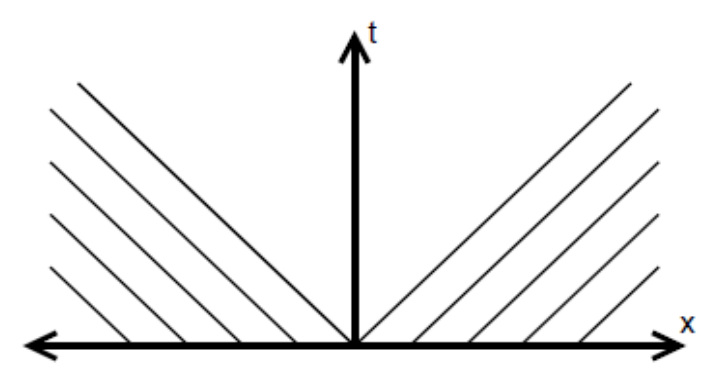

Figure 2: Diagram showing the characteristic curves with a gap, implying non unique solution.

The figure 2 interprets that there is no unique solution for the above problem formulation . Instead the problem or the gap be filled by a number of curves. These curves can be used to fill the gap and multiple solutions can be used. However an additional constraint also called as entropy is added as shown $\mathrm{u}_{\mathrm{t}}(\mathrm{x}, \mathrm{t})+\mathrm{u}(\mathrm{x}, \mathrm{t}) \mathrm{u}_{\mathrm{x}}(\mathrm{x}, \mathrm{t})=\mathrm{eu}_{\mathrm{xx}}(\mathrm{x}, \mathrm{t})$

For conversion of the equation into a hyperbolic ${ }^{[4]}$ one the viscosity term $\epsilon \mathrm{u}_{\mathrm{xx}}(\mathrm{x}, \mathrm{t})$ is included which converts the given equation into a hyberbolic one with a unique solution. This characteristics will introduce a rarefaction of characteristics in the empty gap as shown in the figure below.

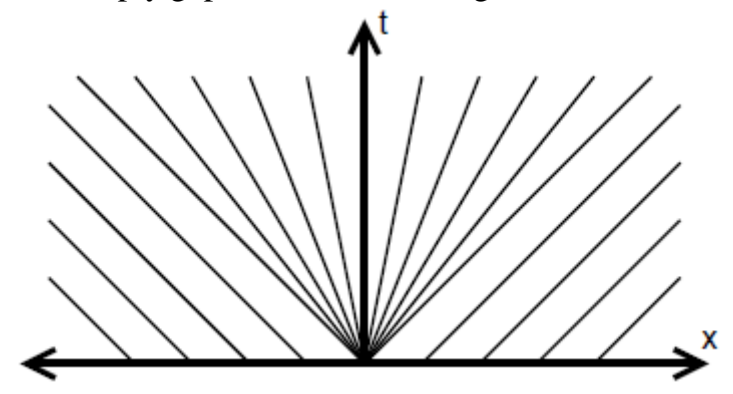

Figure 3. Characteristic curve with refraction wave implying unique solution.

Another scheme which can be implemented is the Upwind scheme. When implementing special attention has to be paid on how to calculate $\Phi_{i j}^{m}$. For simplifying the problem the one dimensional problem can be considered

$$
\begin{aligned}
& D_{z}^{0} U_{i}=\frac{U_{i+1}-U_{i-1}}{2 \Delta x} \ldots(24) \\
& D_{z}^{+} U_{i}=\frac{U_{i+1}-U_{i}}{\Delta x} \ldots(25) \\
& D_{x}^{-} U_{i}=\frac{U_{i}-U_{i-1}}{\Delta x} \ldots(26)
\end{aligned}
$$

These are known as central difference, forward difference and backward difference respectively. In order to select any one of these let us consider one dimensional problem.

$$
U_{t}(x, t)+U_{z}(x, t)=0 . .(27)
$$

$$
\mathrm{U}(\mathrm{x}, 0)=\mathrm{f}(\mathrm{x}) . .(28)
$$


Where the exact solution is $\mathrm{u}(\mathrm{x}, \mathrm{t})=\mathrm{f}(\mathrm{x}-\mathrm{t})=\mathrm{u}(\mathrm{x}-\mathrm{t}, 0)$ and characteristics curves have the slope 1 . Now considering a point $\mathrm{A}$ on the characteristics curve $\mathrm{q}$. The value at $\mathrm{A}$ can be tracked back along $\mathrm{q}$ to a point $\mathrm{B}$, where $t=0$. Hence $B$ is in domain of dependence of Ai.e A depends on $B$. The information flow from A to $B$ during the curve propagation.

So an approximate scheme has to be used that propagates the information in that corresponding direction. In the illustration used information flows from the left to right. Therefore we use backward difference. In general information may flow from the left to right or from the backward to forward direction if needed. be used

In order to send the information on both sides i.e forward and backward the following equations has to

$\frac{u_{i}^{n+1}-u_{i}^{n}}{\Delta t}=-\left(\max \left(a_{i}, 0\right) D_{x}^{-} u_{i}^{n}+\min \left(\alpha_{i}, 0\right) D_{z}^{+} u_{i-1}^{n}\right)$.

Or equivalently it can be written as

$u_{i}^{n+1}=u_{i}^{n}-\frac{\Delta t}{\Delta x}\left(\frac{a_{i}}{2}\left(u_{i+1}^{n}-u_{i-1}^{n}\right)-\frac{\left\|a_{i}\right\|}{2}\left(u_{i+1}^{n}-2 u_{i}^{n}+u_{i-1}^{n}\right)\right.$

where

$u_{i}^{n}=u(i, n) \&$

$a_{i}=a(i)$

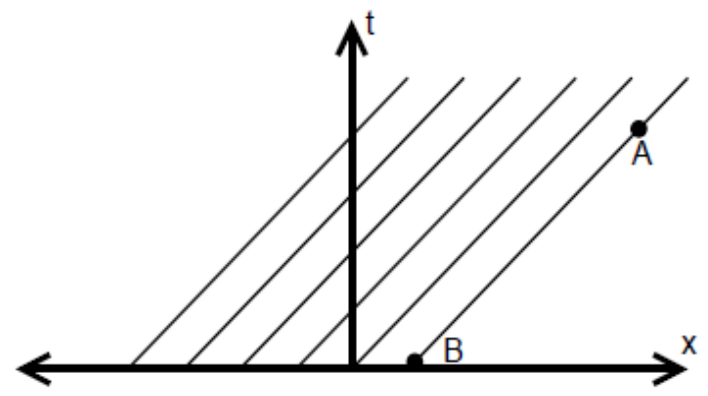

Figure 4 : Characteristic curve showing that $\mathrm{B}$ is in domain of $\mathrm{A}$.

This approach is known as the upwind scheme. Equation 4 is second order term that corresponds to the viscosity term mentioned. Using these upwind characteristics of wave propagation $\mathrm{s}$ and $\nabla \emptyset$ can be calculated for every grid point.

$$
\begin{aligned}
& s . \nabla \emptyset=s \cdot\left(\emptyset_{x^{y}} \emptyset_{y}\right) \\
& =\beta \cdot\left(\left|I^{G}\right|_{z,}\left|I^{G}\right|_{y}\right) \cdot\left(\emptyset_{x^{y}} \emptyset_{y}\right) \\
& \begin{array}{l}
=\beta\left[\max \left(0,\left|\nabla I^{G}\right|_{z}\right) D_{x}^{-} \emptyset+\left[\min \left(0,\left|\nabla I^{G}\right|_{z}\right) D_{x}^{+} \emptyset+\left[\max \left(0,\left|\nabla I^{G}\right|_{y}\right) D_{y}^{+} \emptyset\right.\right.\right. \\
\left.\left.\left.\quad+\left[\min \left(0,\left|\nabla I^{G}\right|_{y}\right) D_{y}^{-} \emptyset\right]\right]\right]\right] . . \text { (31) }
\end{array}
\end{aligned}
$$

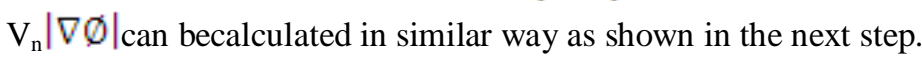

$\mathrm{V}_{\mathrm{n}}|\nabla \emptyset|=\sqrt{\emptyset_{1}^{2}+\emptyset_{2}^{2}}$..

Where

$\emptyset_{1}=\max \left(0, V_{n}\right) D_{x}^{-} \emptyset+\min \left(0, V_{n}\right) D_{x}^{+} . \varnothing$

$\emptyset_{2}=\max \left(0, V_{n}\right) D_{y}^{-} \emptyset+\min \left(0, V_{n}\right) D_{y}^{+} . \emptyset$

In order to preserve the stability of the system while solving the Courants- Friedrichs- Lewy (CFL condition) is applied. The CFL condition takes into consideration the numerical domain of dependence as shown in the figure below. In the particular case the analytical domain of dependence and the dashed line be numerical domain of dependence. 

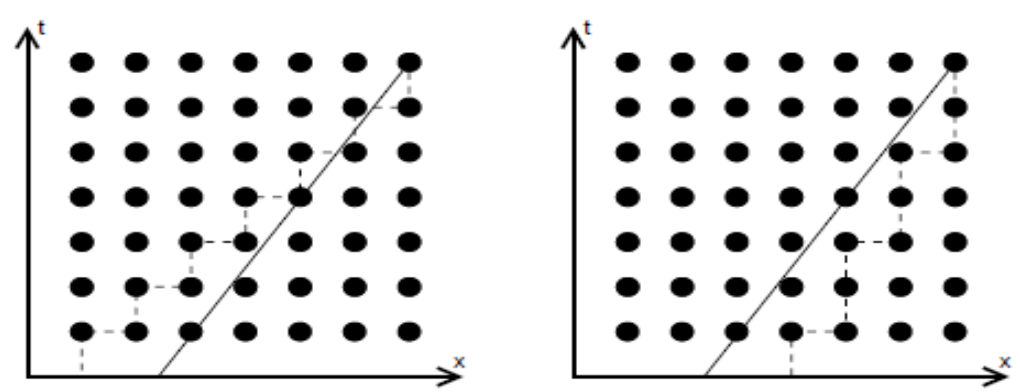

Figure 5. Let the solid line represent the analytical domain of dependence and the dashed line

denote the numerical domain of dependence .In the left figure $\Delta t=1 \Delta t=2$ on the right. On the left the numerical domain of dependence includes the analytical domain of dependence, satisfying the CFL condition .However the CFL condition is violated on the right.

In the first figure the $\Delta t=1$ and $\Delta t=2$ for the second figure. In case of figure 2 the numerical domain of dependence includes the analytical domain of dependence, satisfying the CFL condition. The CFL condition is violated in the second figure.

In case of a CFL condition each point is updated by only its neighboringpoints ,information's should not be moving faster than one grid point per time step. To prevent the appearance of instabilities, the time step is implemented as

$\Delta t=\frac{c}{\max \left\{\left|V_{n}\right|\right\}+\max \{|s|\}} \cdots$

Where $0<c<1$ is some scaling constant set by the user.

The proposed method allows the propagation of the curves in multiple directions by splitting up into two or more curves. The splitted curves can join or merge each other based on the propagation criteria set by the user.

\section{RESULTS}

The method of dual curve propagation was applied on various types of retinal images and the result was analyzed for detection of the exudate formation. The various stages of the diabetic retinopathy are analyzed. The multi wave propagation is used in order to search for multiple exudate formation.

\section{Table I. Input and Exudate Extraction and stage of Diagnosis}

\begin{tabular}{|c|c|c|c|}
\hline Sl. No & $\begin{array}{l}\text { Input Retinal } \\
\text { Image }\end{array}$ & $\begin{array}{l}\text { Exudate } \\
\text { Extraction }\end{array}$ & Stage of Diagnosis \\
\hline 1. & & $\therefore$ & $\begin{array}{l}\text { Background } \\
\text { Diabetic } \\
\text { Retinopathy (BDR) }\end{array}$ \\
\hline 2. & & & $\begin{array}{l}\text { Proliferative } \\
\text { Diabetic } \\
\text { Retinopathy (PDR) }\end{array}$ \\
\hline 3. & & $\phi$ & $\begin{array}{l}\text { Severe Diabetic } \\
\text { Retinopathy (SDR) }\end{array}$ \\
\hline
\end{tabular}

Table 1 shows three stages of diabetic retina proliferation in human eye. The stage 1 is Background Diabetic retinopathy(BDR) where the retinal vessels develop the initial blood clot formation. The stage 2 shows the second stage Proliferative diabetic Retinopathy (PDR) where the blood vessels of the retina starts developing 
the swelling. The last stage is severe Diabetic Retinopathy (SDR) where the entire retinas and the other blood vessels suffers severe diabetic retinopathy and ultimately leads to blindness.

\section{CONCLUSION}

The level set segmentation of multiple wave propagation segments the retinal images and also detects the multiple exudate formation. The proposed method hence helps in early diagnosis of the diabetic retina, when the initial retinal blood clots starts to form in the retina. The proposed method is independent of any kind of inhomogeneity's in the input image.

\section{REFERENCES}

[1] Normalized cuts and image segmentation.Author(s): JianboShi ,Malik, JIEEE Transactions onPattern Analysis and Machine Intelligence, Date of Publication: Aug 2000.Volume: 22 , Issue: 8 Page(s): $888 \quad-905$.

[2] Effective Level Set Image Segmentation With a Kernel Induced Data Term,Ben Salah, M. Ben AyedI., Mitiche, A.Date of Publication: Jan. 2010.Image Processing, IEEE Transactions onImage Processing. Volume: 19 , Issue: 1 .Page(s): 220 - 232

[3] Level Set Segmentation With Multiple Regions .Brox, T.Weickert, J , IEEE Transactions on Image Processing . Date of Publication: Oct. 2006. Volume: 15, Issue: 10, Page(s): 3213- 3218

[4] Distance Regularized Level Set Evolution and Its Application to Image Segmentation. Chunming Li,ChenyangXu ;ChangfengGui ; Fox, M.DIEEE Transactions on Image

[5] Product Type: Journals \& Magazines

[6] Canny Operator Based Level Set Segmentation Algorithm for Medical Images, Xujia Qin, JionghuiJiang ;Weihong Wang ; Fan Zhang. The 1st International Conference onBioinformatics and Biomedical Engineering, 2007. ICBBE 2007Date of Conference: 6-8 July 2007.Page(s): 892-895.

[7] Automatic initialization of level set segmentation for application to optic disc margin identification.Echegaray, S. ,Soliz, P. ; Luo, W.. 22nd IEEE International Symposium onComputer-Based Medical Systems, 2009.CBMS 2009. Date of Conference: 2-5 Aug. 2009. Page(s): 1 - 4

[8] Phase-Based Level Set Segmentation of Ultrasound Images. Belaid, A, Boukerroui, D. ;Maingourd, Y. ; Lerallut, J.-F. IEEE Transactions onInformation Technology in Biomedicine,Date of Publication: Jan. 2011,Volume: 15 , Issue: 1. Page(s): 138 - 147

[9] Neighbor-constrained segmentation with level set based 3-D deformable models, Jing Yang, Staib, L.H. , Duncan, J.S. IEEE Transactions onMedical Imaging,Date of Publication: Aug. 2004, Volume: 23 , Issue: 8 . Page(s): 940 $-948$

[10] Automatic segmentation of liver blood vessels using level set method. Daejeon Jong Won Park International Conference onAudio, Language and Image Processing, 2008.ICALIP 2008. Date of Conference: 7-9 July 2008., Page(s): 1718 1720

[11] Space-time segmentation using level set active contours applied to myocardial gated SPECT. Debreuve, E. ,Barlaud, M. ; Aubert, G. ; Laurette, I. ; Darcourt, J. IEEE Transactions onMedical Imaging, Date of Publication: July 2001.Volume: 20 , Issue: 7 .Page(s): 643 - 659 